\title{
A 6-year-old boy with Sulzberger and Garbe dermatosis: a case report and literature review
}

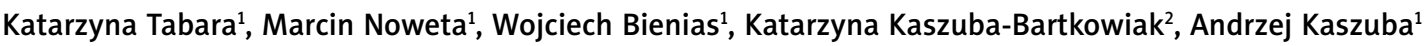 \\ 1Department of Dermatology, Paediatric Dermatology and Dermatological Oncology, Medical University of Lodz, Poland \\ Head of Department: Prof. Andrzej Kaszuba MD, PhD \\ 2Department of Ophthalmology and Visual Rehabilitation, Medical University of Lodz, Poland \\ Head of Department: Prof. Roman Goś MD, PhD
}

Postep Derm Alergol 2013; XXX, 6: 403-408

DOI: $10.5114 /$ pdia.2013.39440

\begin{abstract}
Sulzberger-Garbe disease was described in 1937. Not more than a hundred of cases of the disease have been reported in the literature. Despite a quite specific picture, there are no features that could undeniably be attributed to this particular disease entity. Discoid exudative and lichenoid lesions are main lesions in this dermatosis. The disease is associated with severe pruritus. The lesions are located on the trunk and extremities as well as the genitals. Eosinophilia is frequently found in the course of the disease. Sulzberger-Garbe dermatosis has been diagnosed mainly in middle-aged males of Jewish origin but it can occur in both sexes at any age. Good therapeutic response to systemic corticosteroids has been observed. It is still controversial whether the disease should be classified as an independent clinical entity.
\end{abstract}

Key words: Sulzberger and Garbe dermatosis, distinctive exudative discoid and lichenoid chronic dermatosis, oidoid disease.

\section{Introduction}

Sulzberger-Garbe dermatosis was first described in 1937 based on observations performed on a group of 9 patients treated in the Skin and Cancer Clinic in New York. The name of the disease derives from the names of two physicians: Marion B. Sulzberger and William Garbe. Doctor Sulzberger observed the first cases of the disease in 1927, while practising in Zurich at a department managed by professor Bruno Bloch, and then in 1929 in Wroctaw - at a department ran at that time by professor Joseph Jadasson. Observations of other patients with similar skin disorders were performed together with doctor Garbe in New York [1].

Currently, not more than a hundred of reported cases can be found in the literature. Moreover, most of the reports came from the area of North America in the middle of the $20^{\text {th }}$ century. Despite a quite typical picture of the disease, it still does not show features, which could be undeniably attributed solely to this particular entity. There are no diagnostic tests unequivocally directing the diagnostician at the diagnosis, either. As a result, con- troversies over the legitimacy of distinguishing it as an independent clinical entity continue.

Skin lesions occurring in the course of the disease are mainly discoid exudative ones and are associated with lichenification. Pruritus in the Sulzberger and Garbe dermatosis is typically very severe. Lesions are located on the trunk and extremities as well as in the genital areas. Blood eosinophilia is common in the course of the disease. The Sulzberger and Garbe disease has been diagnosed mainly in middle-aged males of Jewish origin but it may occur in both sexes at any age. Differential diagnosis usually includes mycosis fungoides and other cutaneous lymphomas, allergic contact dermatitis, Duhring's disease, atopic dermatitis, lichen planus as well as nummular eczema and prurigo nodularis.

The case of a 6-year-old boy with the suspicion of Sulzberger and Garbe dermatosis, who was hospitalized at the Department of Dermatology, Paediatric Dermatology and Dermatological Oncology, Medical University in Lodz and a review of the literature concerning the presented rare disease are discussed below in this study.

Address for correspondence: Prof. Andrzej Kaszuba MD, PhD, Department of Dermatology, Pediatric Dermatology and Dermatological Oncology, Medical University of Lodz, 1/5 Kniaziewicza St, 91-347 Lodz, Poland, phone: +48 42651 10 72, e-mail: andrzej.kaszuba@umed.lodz.pl Received: 7.01.2013, accepted: 7.11.2013. 


\section{Case report}

A 6-year-old boy with the initial diagnosis of atopic dermatitis was admitted to the Department of Dermatology at the beginning of March 2012. The boy had hitherto developed normally, achieved weight and height proper for his age. He was born at term, the delivery was spontaneous and he was given 10 points according to the Apgar test. The boy got all vaccinations according to the regular vaccination schedule; in the past he underwent upper respiratory tract infections several times and suffered from no other severe diseases. Widespread skin lesions that increased on the trunk and extremities had been observed in the patient for four months before he was admitted to hospital. According his mother's relation, the first lesion appeared in the left thigh 2 years before the admission and was treated with local corticosteroids ordered by the paediatrician. Shortly before the admission more round and oval skin eruptions, which were partly exudative and with a diameter up to a few centimetres appeared in the boy. In December 2011 a mycological examination was performed - both direct smear and culture went negative. In the middle of February 2012 the child was hospitalized in the pediatric department of infectious diseases, where intravenous antibiotic (Cefuroxime) and antifungal drug (Fluconazole) orally were administered. In the topical treatment ointment containing diflucortolone with isoconazole was used. Ambroxol, diosmectite, dimetindene, clemastine, rehydration solutions, probiotics and, locally: octenidine with phenoxyethanol, gentian violet and mupirocin were also applied. The treatment resulted in reducing the oedema severity and redness of the skin around the lesions, yet new skin lesions appeared during the hospitalization. In the material obtained for mycolog-

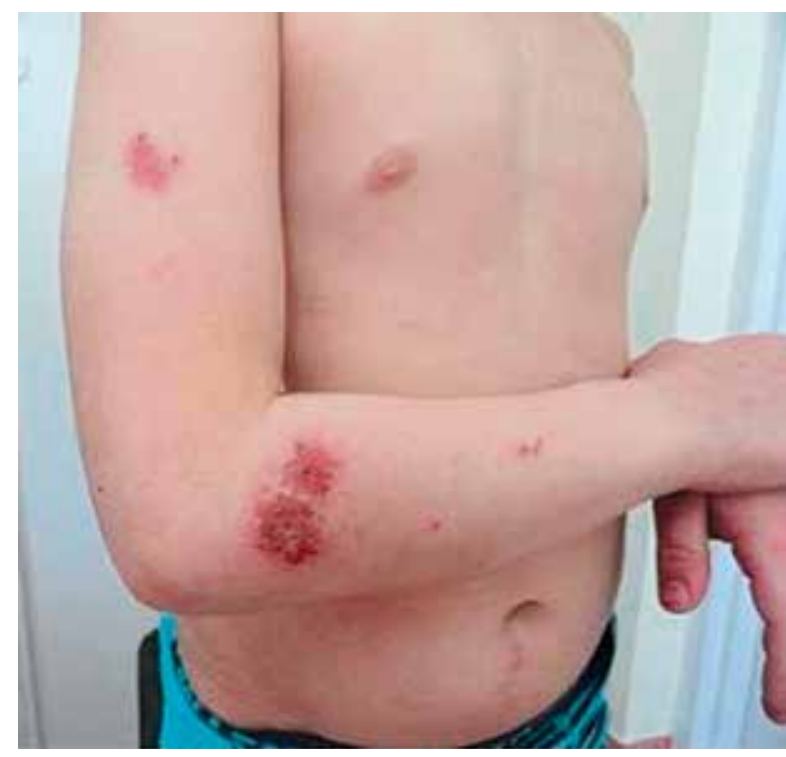

Figure 1. Exudative discoid lesions on the right upper limb on admission ical and bacteriological tests, no microorganism cultures were observed. Due to lack of noticeable improvement the patient was admitted to the department of dermatology.

On admission to the Department of Dermatology, Peadiatric Dermatology and Dermatological Oncology, the boy's skin was dry with exfoliation spots, papular and vesicular eruption developed at his face; it was papular in the interscapular region, lichenoid in the lower and upper extremities as well as in the lateral surface of the trunk: numerous discoid, exudative, dark grey-brown, eminent lichenoid lesions of 4-10 cm in diameter were observed, which appeared clearly separated from the surrounding, partially covered with eschars and without any tendency to merge (Figures 1-3, 4 A, 5 A). Slight redness of the upper palatine arch and dental caries were noted in the physical examination.

Laboratory tests revealed severe eosinophilia; Peripheral Blood Smear: segmented leukocytes - 23\%, eosinophils - 19\%, lymphocytes - 50\%, monocytes - $8 \%$, additionally significant microcytosis and presence of reactive lymphocytes were noted. The general count of leukocytes, erythrocytes and thrombocytes was normal. Biochemical tests revealed normal levels of aspartate transaminase (AST), alanine transaminase (ALT), creatinine and electrolytes. No aberrations in the general urine examination were found and cello-tape anal swab for Enterobius vermicularis was negative. Total concentration of immunoglobulin E was elevated - $330 \mathrm{lU} / \mathrm{ml}$ $(\mathrm{N}$ : <90). Due to herpetic character of the facial exanthem, determination of antibodies was carried out, which showed an elevated level of IgG anti-HSV - 1.8 (N: < 1.1) and a normal level of IgM anti-HSV - 0.9 (N: < 0.9). The

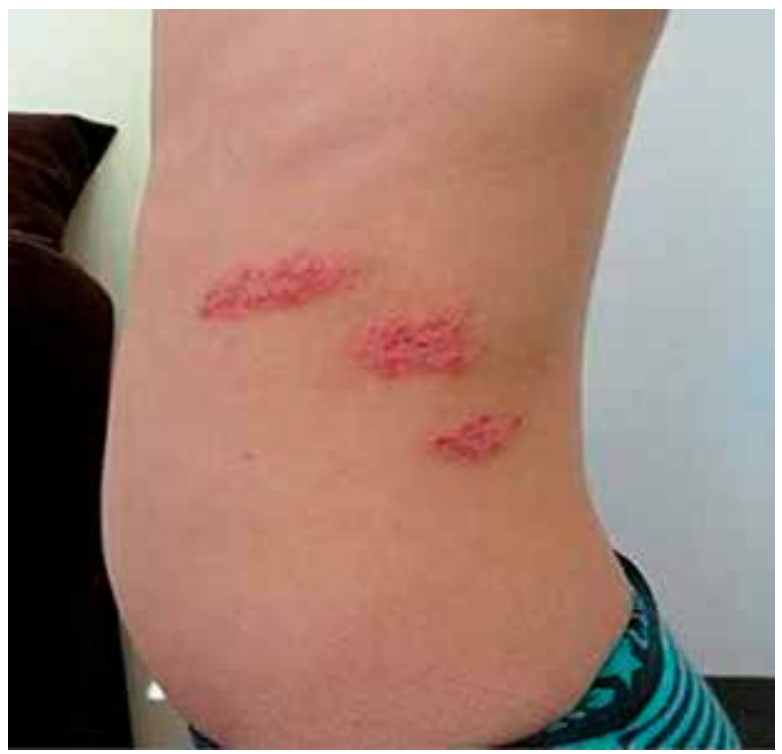

Figure 2. Exudative lichenoid oval lesions on the trunk on admission 


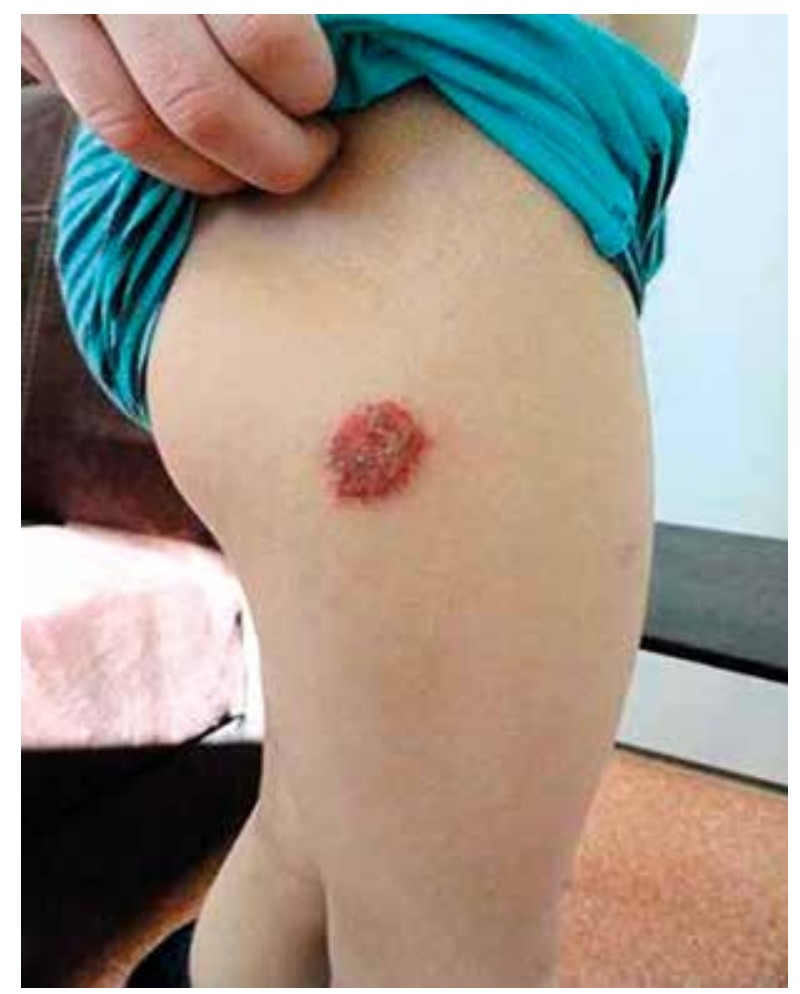

Figure 3. Exudative discoid lesion on the right thigh on admission

boy's mother did not agree for the biopsy and histopathological examination of the skin lesions to be performed.

In the course of hospitalization the patient reported severe pruritus, scratched himself very intensively, as a result of which $1^{\text {st }}$ generation antihistamine medications (clemastine, hydroxyzine, promethazine) were administered together with antibiotic therapy (spiramycin) and externally applied preparations: 2\% gentian violet, boric acid with phenol, resorcinol and rosaniline solution, gentamycine $(0.08)$ and prednisolone (0.5) cream for skin lesions on the trunk and extremities as well as ichthyol paste and vitamin cream for the face. On day 5 of hospitalization one ampoule of betamethasone dipropionate with bethamethasone disodium phosphate was administered intramuscularly. On day 9 of hospitalization the pruritus resolved almost completely, the exudative lesions on the trunk and extremities were in the healing phase, lesions on face and back became pale and tended to decrease. On day 14 of hospitalization the boy was discharged from the hospital in good general condition (Figures $4 \mathrm{~B}$ and $5 \mathrm{~B}$ ). On the day of discharge remitting redbrown erythematous and exfoliative, nonexudative foci remained on the skin of the trunk and extremities, most lesions flattened into the level of the healthy skin. The patient was discharged with a recommendation to take antihistamine drugs (desloratadine in
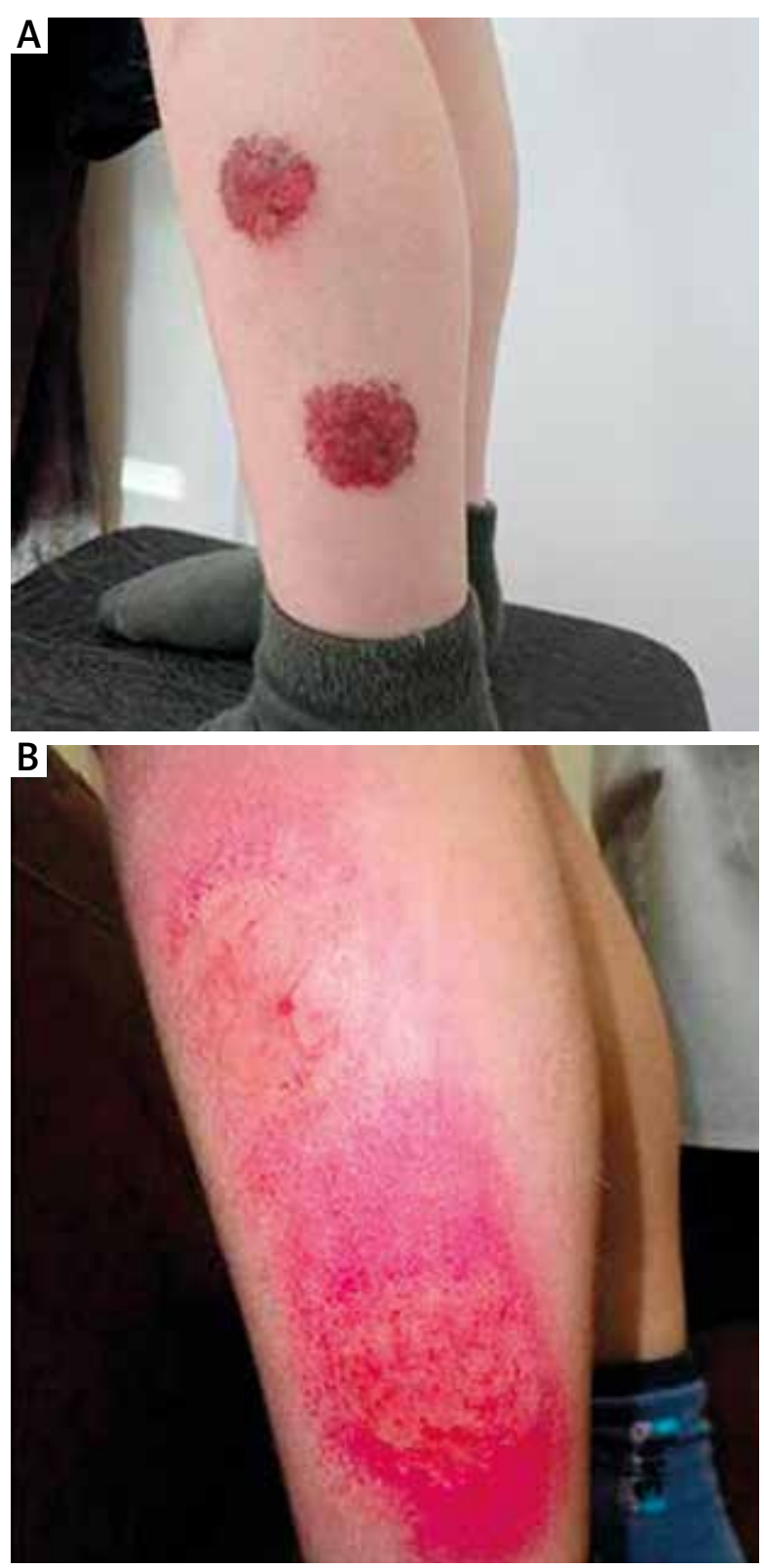

Figure 4. A - Exudative discoid lesions on the left calf on admission. $\mathbf{B}$ - The same lesions in the healing phase at the discharge

the morning, clemastine in the evening) for 7 days and externally apply the prescribed medications: vitamin cream for face and gentamycine with prednisolone creams for the lesions on the trunk and extremities.

\section{Discussion}

As it was pointed out before, there are no typical clinical and histopathological features that would make it possible to diagnose Sulzberger and Garbe dermatosis. In 1979, Sulzberger determined several specific features, 

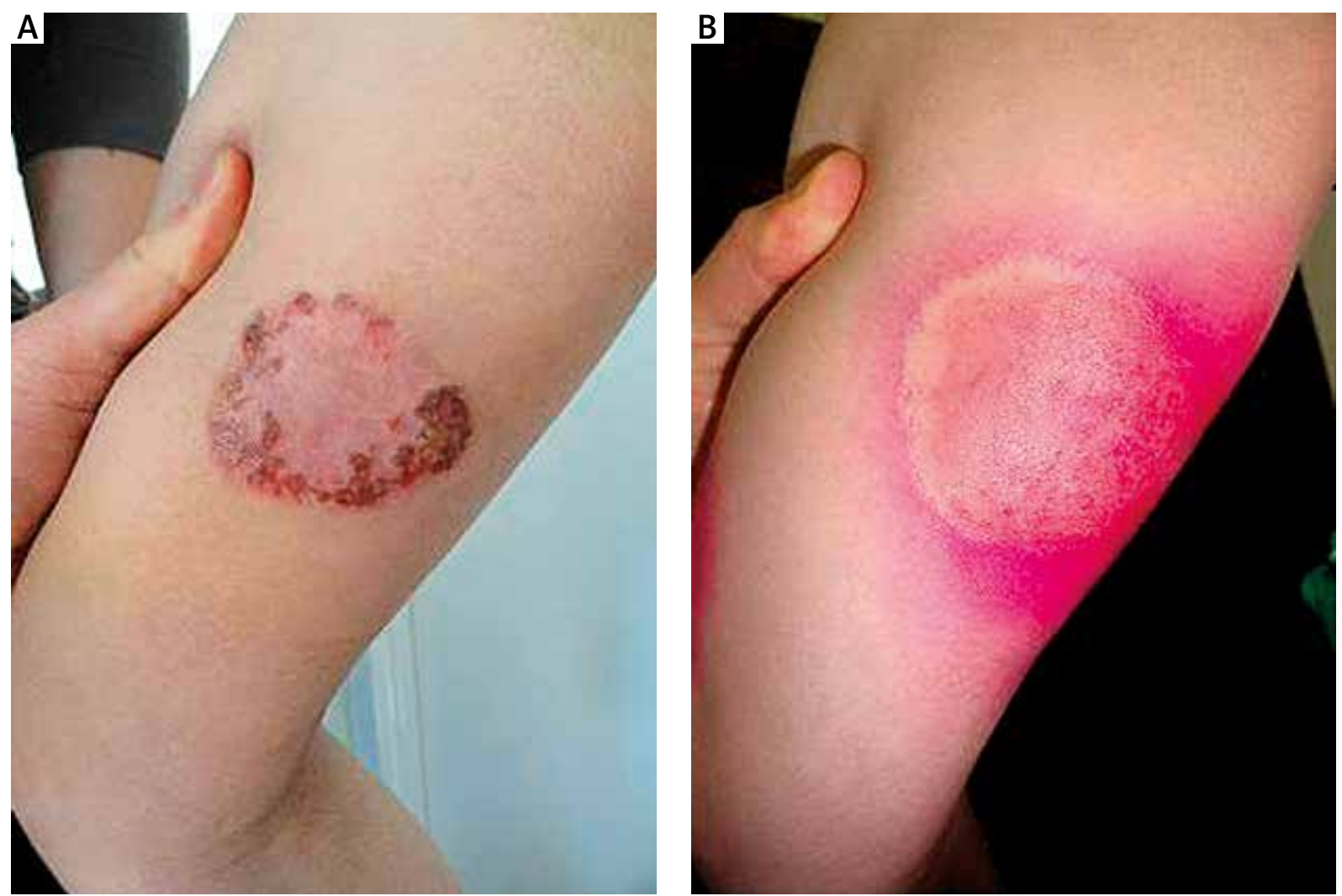

Figure 5. A - Large discoid lesion on the inner surface of the right thigh on admission. B - The same lesion in the healing phase at the discharge

Table 1. Characteristic features of Sulzberger and Garbe dermatosis [1]

- Predilection for the disease among middle-aged males (especially of Jewish origin)

- Before establishing the proper diagnosis other diseases are frequently considered in the diagnostic process

- The skin lesions typically evolve from elevated discoid, exudative to lichenoid ones and vice versa

- Lesions are fairly common on the genitals (especially on penis in males)

- Small, round or oval, oedematous exanthems that imitate urticaria may occur in the phase of spontaneous regression

- Eosinophilia may occur in the peripheral blood

- Mild endothelium swelling and perivascular infiltrations that consist mainly of lymphocytes, granulocytes, eosinophiles and plasmatic cells are frequently observed in the histopathological examination

which may direct the diagnostician towards the diagnosis (Table 1). The author emphasized the predilection of the disease occurrence in middle-aged males (especially of Jewish origin). The skin lesions should typically evolve from eminent discoid, exudative to lichenoid ones and vice versa; in the phase of spontaneous withdrawal small, round or oval, oedematous exanthems may occur, which resemble urticaria. Lesions are fairly common on the genitals. Additional tests may show eosinophilia in the peripheral blood, while in histopathological examination, presence of mild endothelial oedema and perivascular infiltrations mainly consisting of lymphocytes, granulocytes, eosinophiles and plasmatic cells is frequent. The author also mentioned that before establishing the di- agnosis in the diagnostic process other more frequent diseases should be considered [1]. Other features, which were not described by Sulzberger definitely include the occurrence of severe pruritus and good therapeutic reaction to systemic corticosteroids [2].

Undoubtedly, the 6-year-old patient treated in the Department of Dermatology did not satisfy the criteria of age and ethnic origin imposed by Sulzberger. The patient described in this study is probably one of the rare cases of children in whom this disease was suspected. In 1992, Jansen et al. described a case of a 7-year-old girl with skin lesions characterized by a similar morphology, severe pruritus and eosinophilia in peripheral blood, who was effectively treated with corticosteroids administered orally [3]. 
Most cases of the disease were observed between the 1930 s and the 1950 s in the Skin and Cancer Clinic in New York (under the authority of the Columbia University at that time), where Sulzberger and Garbe were practising. A lot of Jewish patients were treated in the Clinic at that time. According to doctor Samuel B. Frank, who also worked in the Clinic, even the information for the patients in the hospital corridors were written both in English and Yiddish. This might be the source of the conviction that the disease occurs mostly in Jewish people [4]. However, the disease was later diagnosed in Europe in patients of non-Jewish background $[5,6]$ and in one Indian patient as well [7]. Nevertheless, the fact of a scarcer occurrence of the disease outside the North America is still difficult to explain [8].

The presently reported boy did not develop any lesions on his penis or scrotum, either. In the course of the disease such lesions may occur later than the other ones and may last longer, in spite of regression of the exanthem on the trunk and extremities [6]. Reports of patients with the suspicion of Sulzberger and Garbe dermatosis who did not develop lesions on the genitalia can also be found in the literature $[5,8]$. Due to this criterion, the incidence of the disease in women may be underestimated [4].

Quite typical discoid, exudative lesions occurred in the studied child and evolved in the course of the treatment; however, it was difficult to assess if they also changed their nature before applying the pharmacotherapy due to lack of precise data in the previous medical documentation of the patient. The primary exanthem should typically be a papule [9].

The reported boy also suffered from intense pruritus. Sulzberger emphasized that pruritus experienced by patients he described seemed the most severe one he had ever observed. The patients were often qualified as nervous and easily falling in the depressive mood, which obviously can be explained by the occurrence of severe pruritus [1].

Quite remarkable eosinophilia in the peripheral blood was also observed in the patient, but such a condition can also occur in the course of numerous allergic, parasitic and neoplastic diseases, collagenases as well as in many other diseases.

Typically, mild endothelium swelling and perivascular infiltrations consisting mainly of lymphocytes, granulocytes, eosinophils and plasmatic cells have been observed in microscopic examination of the skin lesions [1]. Spongiosis and parakeratosis have also been quite frequently visualized [2]. In 1984, Stevens and Ackerman re-examined the histopathological specimens from patients treated in New York for Sulzberger and Garbe dermatosis and they found no characteristic features, which would make it possible to differentiate between this disease and nummular eczema on the basis of microscopic examination of the cutaneous lesions [10]. It is currently thought that specific features other than occurrence of eosinophils in some cases cannot be found in histopathological examinations [9]. There are only two reports on immunofluorescence tests of skin samples in patients with the suspicion of Sulzberger and Garbe dermatosis in the literature. In the first case, occurrence of a slight amount of C3 complement was found in the area of the basement membrane [5], whereas in the other one, deposits of C3 at the dermoepidermal junction and deposits of IgM and C3 in the vascular walls in the upper layers of the dermis were detected. The described image could therefore represent lichen planus [2].

Typically, the Sulzberger and Garbe dermatosis patients responded well to the treatment even with small doses of systemic corticosteroids, in most cases administered orally [1]. In the child hospitalized at the Department of Dermatology, Paediatric Dermatology and Dermatological Oncology, a prolonged-release corticosteroid was applied intramuscularly, as a result of which the severity of pruritus and size of skin lesions decreased. Reports of two cases of Sulzberger and Garbe dermatosis in patients who initially did not respond to corticosteroids and in whom azathioprine was successfully administered have been presented in the literature [5]. Skin lesions in this disease seem to be resistant to topical corticosteroids. Beneficial effects of narrow band UVB radiations have also been reported [9]. Sulzberger recommended his patients to move to a different climate, which was supposed to considerably improve the condition of the skin [1]. Such recommendation usually results in improvement in patients with atopic diseases. Unfortunately, no observations and studies have been performed in this respect so far. Interestingly, an increased level of immunoglobulin E was noted in the child hospitalized at our Department, which may suggest the occurrence of atopy.

The diagnosis of Sulzberger and Garbe disease can be established after excluding other more frequent diseases; considering other diagnoses before reaching the final one was also classified as a diagnostic criterion by Sulzberger. Tinea cutis glabrae (ruled out twice in mycological examinations) and atopic dermatitis were initially suspected in the child; the character of skin lesions could also indicate nummular eczema. The patients in the literature usually had a suspicion of mycosis fungoides or other subtype of skin lymphoma or allergic contact dermatitis, slightly less frequently Duhring's disease, atopic skin dermatitis, psoriasis or lichen planus; the differential diagnosis less frequently included seborrheic dermatitis, parapsoriasis, tinea cutis glabrae and dermatophytid reaction, drug rashes, pityriasis rubra pilaris or nummular eczema [1]. Similarities between Sulzberger and Garbe dermatosis and mycosis fungoides include severe pruritus and the peak of the incidence occurring in middle-aged patients. Skin lesions in mycosis fungoides usually are not exudative on the surface in the initial and infiltrative phases of the disease, lichenoid 
lesions are not observed, either. These entities can be differentiated in histopathological examination, based on the occurrence of lesions on the genitalia and skin folds in flexural areas, evolution of lesion morphology and regression following treatment with systemic corticosteroids. Exudative lesions and improvement after moving to a different climate zone may occur in the course of allergic contact dermatitis, but unlike Sulzberger and Garbe disease, a typical history of contact with allergen is found and patch tests show hypersensitivity. In allergic contact dermatitis, the skin lesions do not typically evolve from eminent discoid, exudative to lichenoid and there is no predilection for the occurrence in the area of the genitalia or the incidence in middle-aged subjects. Nummular eczema is characterized by quite clearly separated erythematous foci, which are covered with papules and bullae or exfoliating epidermis in chronic lesions. The lesions are usually round or oval. Patients also feel pruritus in the course of nummular eczema but it is usually moderate and the lesions hardly ever occupy the genitals. Improvement is observed after topical corticosteroids in nummular eczema, but systemic administration of corticosteroids may in some cases be necessary [1, 9].

\section{Conclusions}

Although the Sulzberger and Garbe dermatosis was first described 75 years ago, this disease is still a source of controversies over its existence as a separate nosological entity. As mentioned before, it is puzzling that most cases were observed in the middle of the $20^{\text {th }}$ century, mainly in North America. Subsequent reports and cases from other regions are scarce. No report from the recent 15 years can be found in the PubMed database. Sulzberger suggested that the decrease in the dermatosis incidence could result from growing popularity of corticosteroids. The author expected that pathological changes characteristic of only this disease would be found as a result of increased use of modern diagnostic techniques. He also suggested that small skin vessels became damaged in the course of the disease [1]. However, no advanced studies have been performed in a large population of patients since the Sulzberger and Garbe disease is currently hardly ever diagnosed. Jansen et al. concluded their report of a 7-year-old girl's case that there were no clinical or histological features, which would make it possible to differentiate between Sulzberger and Garbe dermatosis and nummular eczema [3]. Similarly, in the presently discussed case it was impossible to dispel all the doubts. Rangioletti et al. considered the diagnosis of Sulzberger and Garbe disease as a temporary diagnosis in the course of chronic exudative-discoid and lichenoid lesions [10]. It seems to be the most rational approach to diagnosing this dermatosis.

\section{References}

1. Sulzberger MB. Distinctive exudative discoid and lichenoid chronic dermatosis (Sulzberger and Garbe) re-examined-1978. Br J Dermatol 1979; 100: 13-20.

2. Rongioletti F, Corbella L, Rebora A. Exudative discoid and lichenoid chronic dermatosis (Sulzberger-Garbe) a fictional disease? Int I Dermatol 1989; 28: 40-3.

3. Jansen T, Küppers U, Plewig G. Sulzberger-Garbe exudative discoid and lichenoid chronic dermatosis ("Oid-Oid disease") - reality or fiction? [German]. Hautarzt 1992; 43: 426-31.

4. Frank SB. Exudative discoid and lichenoid chronic dermatitis: does it exist or should it be discarded? Int I Dermatol 1989; 28: 479 .

5. Freeman K, Hewitt M, Warin AP. Two cases of distinctive exudative discoid and lichenoid chronic dermatosis of Sulzberger and Garbe responding to azathioprine. Br J Dermatol 1984; 111: 215-20.

6. Trüeb RM. Exudative discoid and lichenoid chronic dermatosis (Sulzberger-Garbe): presentation with a puzzling penile plaque. J Eur Acad Dermatol Venereol 1994; 3: 411-7.

7. Kanwar AJ, Kaur S, Bansal R, et al. Sulzberger-Garbe Dermatosis in an Indian Patient. Int J Dermatol 1989; 28: 44-5.

8. Rebora A. Sulzberger-Garbe disease in Europe. Int J Dermatol 1989; 28: 22.

9. Bieber T. Inne odmiany zapalenia skóry [Polish]. In: Braun-Falco Dermatologia. Burgdorf WHC, Plewig G, Landthaler HH (eds.). Czelej, Lublin 2011; 450.

10. Stevens DM, Ackerman AB. On the concept of distinctive exudative discoid and lichenoid chronic dermatosis (Sulzberger-Garbe). Am J Dermatopathol 1984; 6: 387-95. 